\title{
Novel biomarker SYT12 may contribute to predicting papillary thyroid cancer outcomes
}

\author{
J Jonklaas ${ }^{\star}, \ddagger \S, 1$, SRK Murthy ${ }^{\ddagger}, 2$, D Liu ${ }^{3}$, J Klubo-Gwiezdzinska ${ }^{4}$, J Krishnan ${ }^{5}$, KD Burman ${ }^{6}$, L \\ Boyle $^{7}, \mathrm{~N} \mathrm{Carrol}^{8}$, E Felger ${ }^{8} \&$ Y P Loh ${ }^{* *}, \S, 2$ \\ ${ }^{1}$ Division of Endocrinology, Georgetown University, Washington, DC 20007, USA \\ ${ }^{2}$ Section on Cellular Neurobiology, Eunice Kennedy Shriver National Institute of Child Health \& Human Development, National \\ Institutes of Health, Bethesda, MD 20892, USA \\ ${ }^{3}$ Division of Intramural Population Health Research, Biostatistics \& Bioinformatics Branch, Eunice Kennedy Shriver National Institute \\ of Child Health \& Human Development, National Institutes of Health, Bethesda, MD 20892, USA \\ ${ }^{4}$ National Institutes of Diabetes \& Digestive \& Kidney Diseases, National Institutes of Health, Bethesda, MD 20892, USA \\ ${ }^{5}$ Department of Pathology, MedStar Washington Hospital Center, Washington, DC 20010, USA \\ ${ }^{6}$ Section of Endocrinology, MedStar Washington Hospital Center, Washington, DC 20010, USA \\ ${ }^{7}$ Department of Surgery, Georgetown University, Washington, DC 20007, USA \\ ${ }^{8}$ Department of Surgery, MedStar Washington Hospital Center, Washington, DC 20010, USA \\ * Author for correspondence: Tel.: +1 202687 2818; Fax: +1 877485 1479; jonklaaj@georgetown.edu \\ ** Author for correspondence: Tel.: +1 (301) 496 3239; Fax: +1 (301) 496 9938; lohp@mail.nih.gov \\ $¥$ Authors contributed equally \\ $\S$ Co-corresponding authors
}

\begin{abstract}
Aim: To investigate biomarkers for predicting papillary thyroid cancer outcomes. Materials \& methods: The expression of biomarkers (ITGA2, SYT12 and CDH3) was studied in a prospective cohort of patients with papillary thyroid cancer. Three outcomes of initial metastases, baseline status and longitudinal status were analyzed and correlated with the biomarkers. Results: SYT12 provided the best prediction of initial metastasis (sensitivity: 72\%; specificity: 54\%). SYT12 had the highest accuracy for predicting longitudinal status (sensitivity: 100\%; specificity: 47\%). The best performance for longitudinal status resulted from combining SYT12 with American Thyroid Association risk stratification, with sensitivity and specificity of 88 and $73 \%$, respectively. Conclusion: SYT12 has some prognostic significance in papillary thyroid cancer. Further validation studies in larger populations are warranted.

Lay abstract: Survival and disease recurrence after diagnosis of papillary thyroid cancer is imperfectly predicted by commonly used 'staging systems' based mostly on the characteristics of the tumor such as size and location of spread. Also, these staging systems are generally designed to predict survival. Other characteristics, such as gene mutations may be helpful in predicting whether thyroid cancer will behave aggressively or not. This study investigates three genes associated with thyroid tumors and shows that expression of one of them, synaptotagmin 12 (SYT12), tends to predict greater progression of disease in patients with papillary thyroid cancer.
\end{abstract}

First draft submitted: 7 July 2017; Accepted for publication: 4 September 2017; Published online: 22 September 2017

Keywords: biomarkers $\bullet$ metastases $\bullet$ papillary thyroid cancer $\bullet$ prognosis $\bullet$ progression $\bullet$ recurrence $\bullet$ SYT12

Differentiated thyroid cancer (DTC) is one of the most rapidly increasing cancers in the USA. For the period 20072011, the largest annual increases in cancer incidence were for cancers of the thyroid $(5.3$ and $4.5 \%$ in men and women, respectively) [1]. Death rates increased by $1.6 \%$ in men and $0.8 \%$ in women, at a time when the death rates from some other malignancies were decreasing. Papillary thyroid cancer (PTC) is the most prevalent histological thyroid cancer subtype, accounting for at least $80 \%$ of all cases [2-4]. DTC prognosis can be estimated based on traditional staging systems that estimate risk of death from thyroid malignancy. According to the seventh edition of the American Joint Cancer Committee/Union Internationale Contre le Cancer staging system (tumor, nodes, metastases [TNM]), patients can be classified as stage I, II, III or IV, with risk of death successively increasing with 
advancing stage [5,6]. Another staging system with excellent performance is the National Thyroid Cancer Treatment Cooperative Study Group staging system (NTCTCSG) in which survival rates for the four successive stages are better separated compared with other staging systems $[2,4,6,7]$.

With the death rates from DTC typically being relatively low, it is generally more useful for clinicians to be able to estimate recurrence risks, rather than risk of death. The American Thyroid Association (ATA) risk stratification system was developed to better define risk of recurrence as low, intermediate or high [5]. Other systems incorporate algorithms to restratify patients once their initial response to their surgery and radioactive iodine (RAI) therapy has been assessed [8]. Despite use of these clinicopathologic staging systems, DTC can often have an unpredictably either less or more aggressive course than had been anticipated.

These challenges to accurate prognostication and confident prediction of aggressive presentation and recurrence in DTC occur because a cascade of genetic, molecular and cellular mechanisms are involved in the transition from differentiated to undifferentiated thyroid tumor [9]. The wide spectrum of DTC behavior has led to a search for molecular markers that may refine risk stratification. Examples of useful markers for PTC are the presence of BRAF V600E mutations, TERT C228T mutations and coexistent BRAF-TERT mutations [10]. MicroRNAs (miRNAs) have also been used as prognostic indicators in DTC [11]. Three miRNAs (miR-146b, miR-221 and miR-222) have consistently been found to be overexpressed in PTC tissue, compared with normal thyroid tissue, and also appear to confer high-risk features.

The staging systems that are discussed above may not, alone, be enough to accurately predict the prognostic outcome of DTC. In this prospective pilot study, we sought to identify molecular targets that would aid in predicting prognosis of PTC, along with the ATA risk stratification system. A set of molecular markers was initially selected based on their differential expression in a training set of PTC tissue samples and finally a set of three gene markers (ITGA2, SYT12 and CDH3) was selected and tested for prognostic significance in an independent PTC cohort. To our knowledge, ITGA2, SYT12 and CDH3 have not been investigated prospectively as biomarkers for predicting disease progression in patients with PTC.

\section{Materials \& methods}

Discovery component

Initial selection of 15 biomarkers

We used gene expression profiling generated by high-throughput platform Gene Expression Omnibus (www.ncbi. nlm.nih.gov/geo) to screen for molecular markers for PTC. Initially, screening of differentially expressed genes in thyroid carcinoma tissues was carried out by manually querying publicly available data at the Gene Expression Omnibus database under the dataset series GSE3678 (www.ncbi.nlm.nih.gov/geo/query/acc.cgi?acc=GSE3678), (samples: GSM85215 thyroid_normal_1, GSM85216 thyroid_normal_2, GSM85217 thyroid_normal_3, GSM85218 thyroid_normal_4, GSM85219 thyroid_normal_5, GSM85220 thyroid_normal_6, GSM85221 thyroid_normal_7, GSM85222 thyroid_tumor_1, GSM85223 thyroid_tumor_2, GSM85224 thyroid_tumor_3, GSM85225 thyroid_tumor_4, GSM85226 thyroid_tumor_5, GSM85227 thyroid_tumor_6 and GSM85228 thyroid_tumor_7). A total of 15 genes were selected (see below). These included genes from this data series whose selection were based on differential expressions between normal and tumor tissue. Other genes of interest based on available laboratory data and primer availability were also chosen.

Use of markers in exploratory or training set of 12 tissue samples

These 15 genes (IPCEF1, FN1, ITGA2, TPO, SYT12, GPMGA, DIO1, CRABP1, STRA6, TFF3, TM7SF4, OTOS, CDH3, DTX4 and TACSTD2) were analyzed for expression by qPCR in our exploratory set of 12 normal and tumor thyroid samples obtained from the Georgetown University Tumor Bank. The details of primers used in the mRNA expression studies and the size of the fragment obtained are shown in Table 1. The annealing temperature was $60^{\circ} \mathrm{C}$. Patient outcomes, including documentation of recurrence and death, were available for a subset of these tumors. Genes that showed greater than twofold differential expression in the thyroid tumors compared with the normal thyroid tissue, or that showed greater than twofold differential expression in tumors from patients who were known to have poor outcomes such as recurrence or death were selected for further analysis in our independent prospective study. A set of three gene markers (ITGA2, SYT12 and CDH3) were selected on the basis of their differential expression and assayed for prognostic significance in the independent cohort. A brief flowchart of the method used in this study for selecting molecular markers for outcomes in PTC is presented in Figure 1. 
Table 1. Details of mRNA expression studies.

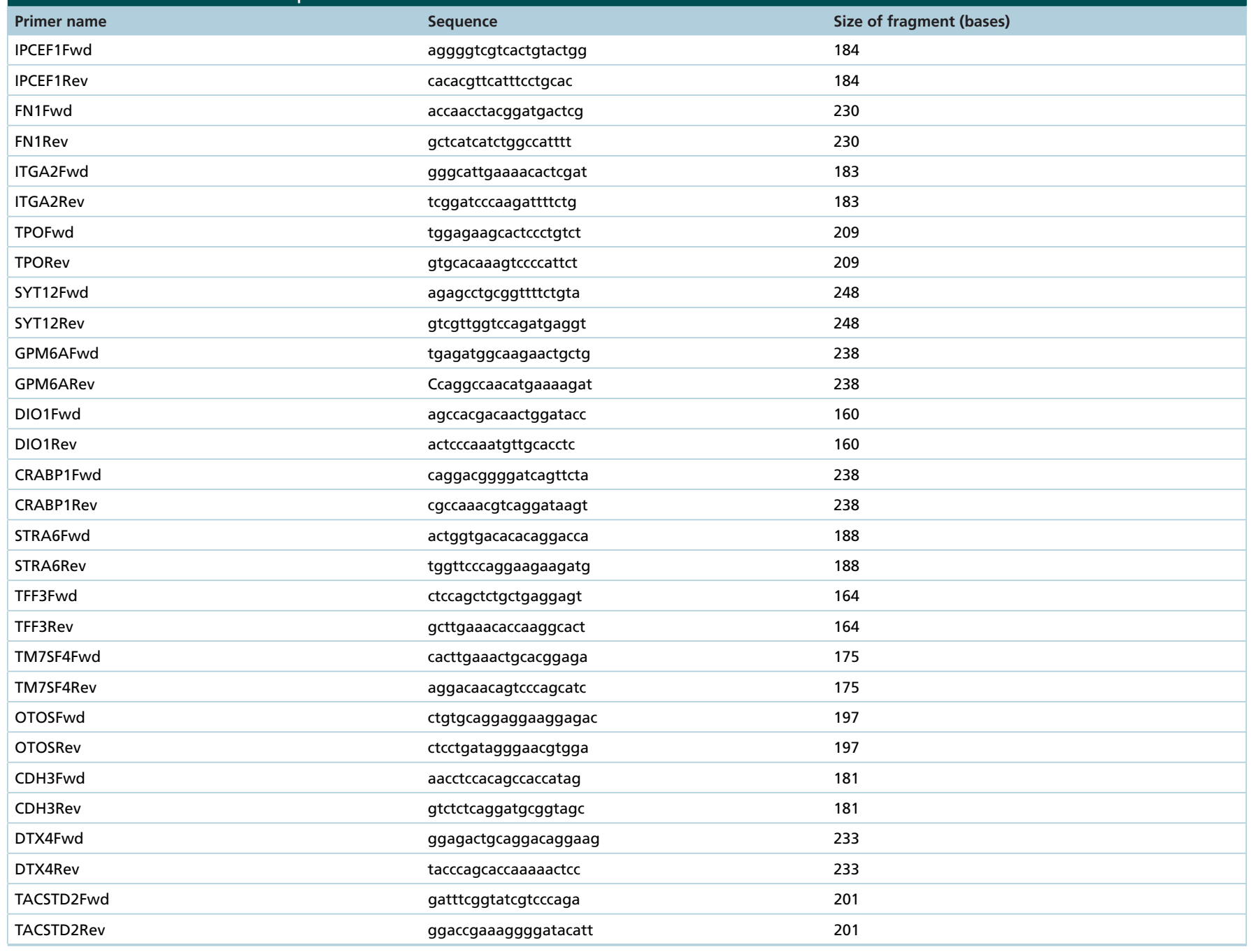

\section{Validation or prospective component}

Design of independent study

In this independent study, we examined biomarkers that would aid in prognosis of PTC, along with the ATA risk stratification system. Patients who had been diagnosed with PTC on the basis of a fine needle aspiration biopsy consistent with PTC (Bethesda category VI), or on the basis of a biopsy that was suspicious for malignancy (Bethesda category V) and felt by the cytopathologist to most likely represent PTC and who were scheduled for surgery at MedStar Washington Hospital Center were recruited for this prospective study. Total thyroidectomy was routinely performed during this time period; central and lateral lymph node dissection was performed at the discretion of the surgeon. Patients were simply recruited sequentially and no patient with PTC was excluded. The study was approved by the Joint Oncology Institutional Review Board at Georgetown University and MedStar Washington Hospital Center. Patients were given information about the study by their surgeon. Interested patients signed a written consent form to allow removal of a small portion of both their tumor and normal thyroid tissue, and also permit review of their medical records using MedStar's Electronic Medical Record System. At the time of their thyroidectomy, approximately 1-gm pieces of tissue were removed from the patient's tumor and from uninvolved thyroid tissue by the study pathologist and rapidly frozen. Frozen tissue was immediately transported to the NIH for RNA extraction. The participant's records were reviewed after their initial treatment with surgery and RAI was complete.

The records were also reviewed on an annual basis for at least 4 years, and in a minority of cases for up to 5 years, to determine whether any additional treatment had been administered and what the patient's disease status was. 
Select thyroid carcinoma data sets in gene expression omnibus

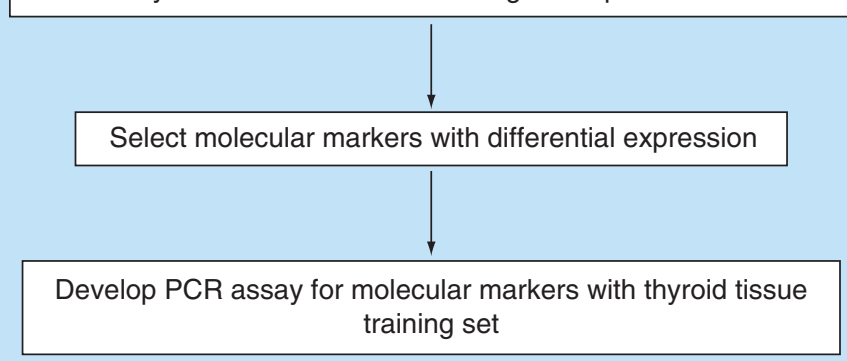

Select molecular markers with differential expression in thyroid tissue training set

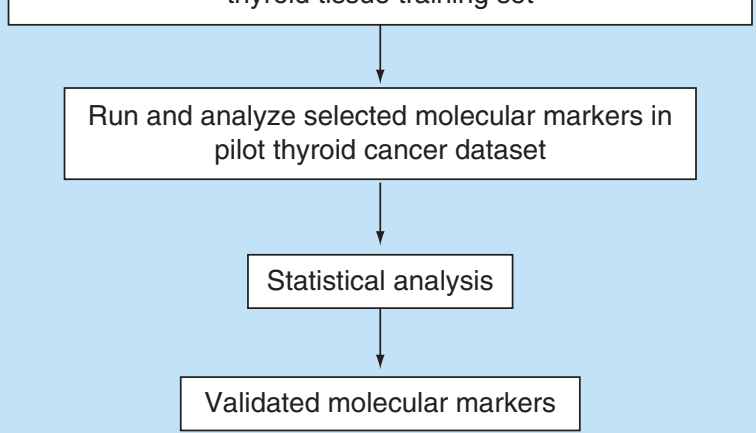

Figure 1. Flowchart of the method of selecting molecular markers for testing in the training and validation studies.

Initial disease status was based on a combination of surgical pathology, results of postoperative RAI scanning and postoperative serum thyroglobulin. Disease status at follow-up was classified as no evidence of disease, biochemical evidence of disease only, regression of disease, stable disease or progression of disease. This status was assigned by one physician based on the results of serum thyroglobulin measurements, thyroglobulin antibody measurements, cervical ultrasonography, 3D imaging (CT scanning, MRI scanning), nuclear medicine studies (RAI whole body scanning and PET scanning) and results of biopsies or additional surgery. A thyroglobulin level of $<0.1-0.5 \mathrm{ng} / \mathrm{ml}$ was considered undetectable, depending on the assay used. A rising thyroglobulin, or a rising thyroglobulin antibody level if the patient had thyroglobulin antibodies present, was defined as 'biochemical disease only' if no structural disease was identified. The physician was blinded to the results of ITGA2, SYT12 and CDH3 mRNA analysis until these results were finalized. The surgical, nuclear medicine and endocrinology care received by patients enrolled in the study was that recommended by their healthcare team at MedStar Washington Hospital Center and was unaffected by study participation.

\section{Characteristics of participants in independent study}

A total of 40 patients were recruited for the independent study during the period 2010-2012. Two patients were ineligible because of a finding of benign thyroid disease and metastatic lung cancer to the thyroid, respectively. Both of these patients had initial biopsies showing suspicious for malignancy rather than PTC. Thus 38 patients were confirmed to have PTC and these patients were monitored for $4-5$ years. The initial demographic, PTC and treatment characteristics of the 38 patients are shown in Table 2. Other tumor characteristics included 66\% with disease-affecting cervical lymph nodes, 39\% with extra-thyroidal extension of their PTC, 63\% with multifocal PTC and $11 \%$ with distant metastases. None of the histologic variants of PTC were minimally invasive follicular variants. All patients received a total thyroidectomy and $92 \%$ received radioiodine (RAI), as was typical for managing DTC at Washington Hospital Center during this time period. The average RAI treatment activity administered was $133 \mathrm{mCi}$ (range: 30-394 mCi). Using the TNM staging system, 61\% of patients had stage I disease, 13\% had stage II disease, $15 \%$ had stage III disease and $11 \%$ had stage IV disease. Using the ATA risk stratification system, 
Table 2. Initial characteristics of patients enrolled in prospective study.

Patient and tumor characteristics $(n=38)$

Continuous variables

\begin{tabular}{|l|}
\hline Age (years) \\
\hline Tumor size (largest focus) $(\mathrm{cm})$ \\
\hline Multifocality: number of foci \\
\hline Number of cervical lymph nodes \\
\hline Treatment activity of radioiodine $(\mathrm{mCi})$ \\
\hline
\end{tabular}

$\begin{array}{lr}\text { Mean } & \text { SD } \\ 45.5 & 13.8\end{array}$

$1.9 \quad 0.7$

$3 \quad 2.9$

$7 \quad 15$

133

Categorical variables

Number Percentage

Sex

- Female 34

- Male

Total thyroidectomy

- Yes

38

100

DTC histology

- Papillary

$27 \quad 71$

- Variants, papillary

11

29

Cervical node involvement

- Yes

- No

$25 \quad 66$

Extrathyroidal extension

- Yes

- No

13

66

34

Multifocality

- Yes

- No

Distant metastases

- Yes

- No

$\begin{array}{ll}34 & 11 \\ & 89\end{array}$

Radioiodine therapy

\section{- Yes}

- No

$\begin{array}{ll}35 & 92 \\ 3 & 8\end{array}$

TNM stage

$-1$

$-11$

- III

- IV

$\begin{array}{ll}23 & 61 \\ 5 & 13 \\ 6 & 15 \\ 4 & 11\end{array}$

\section{NTCTCSG stage}

$\begin{array}{lll}-1 & 22 & 58\end{array}$

$-11$

- III $\quad 9 \quad 24$

-IV $\quad 2 \quad 5$

DTC: Differentiated thyroid cancer; NTCTCSG: National Thyroid Cancer Treatment Cooperative Study Group; TNM: Tumor, nodes, metastases.

$47 \%$ had low-risk disease, $42 \%$ had intermediate-risk disease and $10 \%$ had high-risk disease. Five patients (13\%) within the cohort had positive thyroglobulin antibodies.

$m R N A$ expression studies in 38 resected thyroid tumors from independent cohort

ITGA2, SYT12 and CDH3 mRNAs were determined in resected thyroid tumors as follows.

Normal and tumor tissue samples were homogenized using a polypropylene disposable mortar and pestle (Thermo Fisher Scientific, MA, USA) in TRIzol reagent (Invitrogen, CA, USA) and total RNA was extracted. A total of 


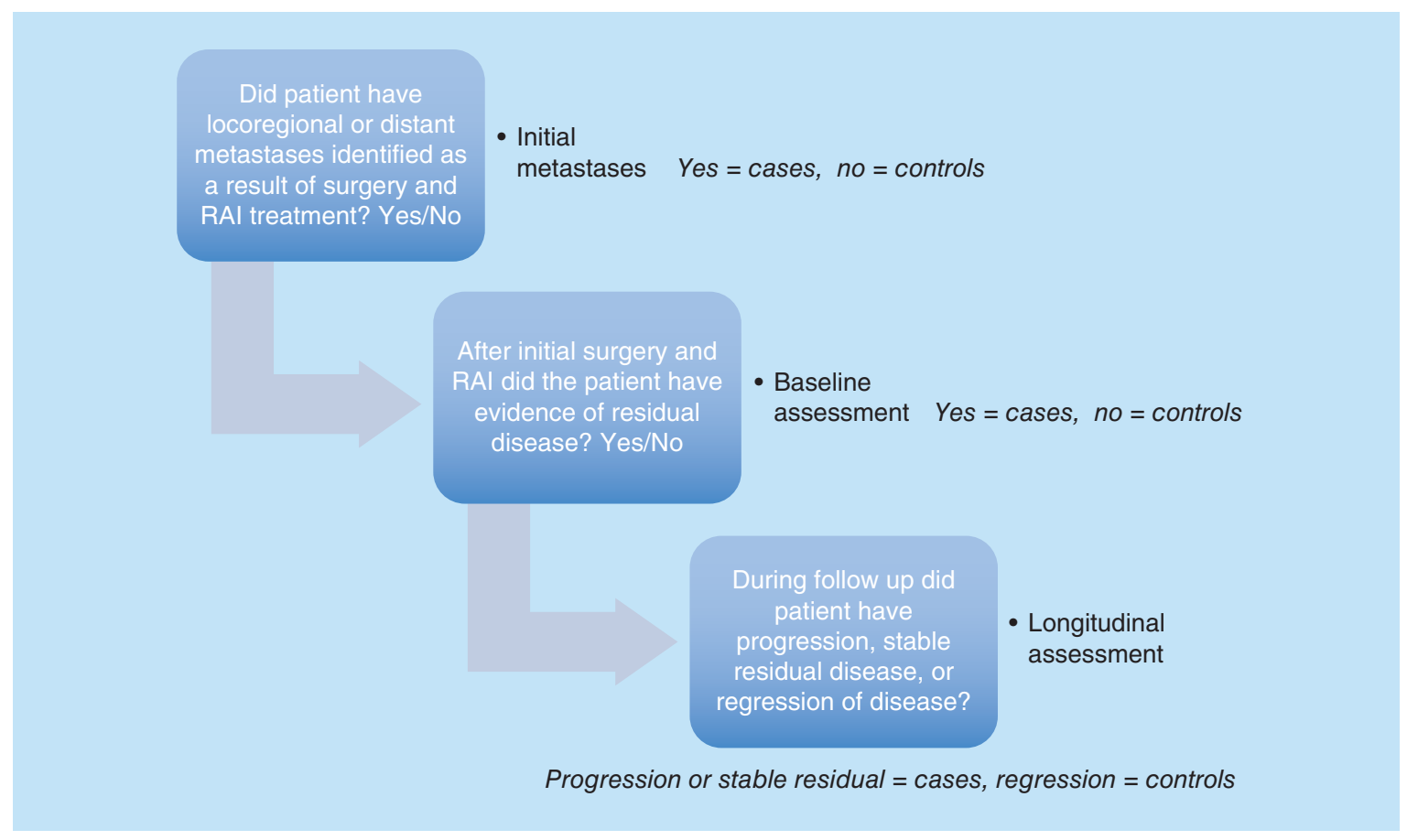

Figure 2. Definition of the dichotomized outcomes of initial metastases, baseline status and longitudinal status used in the prospective cohort.

200 ng of total RNA was reverse-transcribed into complementary DNA using a first strand synthesis kit (Roche, CA, USA) with random hexamers. Complementary DNA generated from the RNA was subjected to real-time quantitative PCR (qPCR) for ITGA2, SYT12 and CDH3 expression using a Fast SYBRxGreen Master Mix PCR kit (Applied Biosystems, CA, USA) under the following cycling conditions: initial denaturation for $5 \mathrm{~min}$ at $95^{\circ} \mathrm{C}$, followed by 40 cycles of $15 \mathrm{~s}$ at $95^{\circ} \mathrm{C}$ and $60 \mathrm{~s}$ at $62^{\circ} \mathrm{C}$. The PCR reaction was followed by a melting curve program $\left(60-95^{\circ} \mathrm{C}\right)$ with a heating rate of $0.1^{\circ} \mathrm{C}$ per second and a continuous fluorescence measurement and a cooling program at $40^{\circ} \mathrm{C}$. Reactions were performed using an ABI PRISM 7500 Sequence Detector (Applied Biosystems). Fluorescence signals were analyzed using SDS 1.9.1 software (Applied Biosystems). The primers used are described earlier.

The relative amounts of biomarker mRNAs (ITGA2, SYT12 and CDH3) were normalized to the housekeeping gene 18S. Fold changes were calculated using the equation $2-\Delta \Delta \mathrm{C}_{\mathrm{T}}, \Delta \Delta \mathrm{C}_{\mathrm{T}}=\left[\mathrm{C}_{\mathrm{T}}\right.$ (biomarker gene) $-\mathrm{C}_{\mathrm{T}}$ (18S) $]$ test $-\left[\mathrm{C}_{\mathrm{T}}\right.$ (biomarker) $\left.-\mathrm{C}_{\mathrm{T}}(18 \mathrm{~S})\right]$ calibrator. The relative expression level of the biomarkers in each sample was evaluated as the relative fold change in $\log 2$ scale. The $\mathrm{T} / \mathrm{N}$ ratio was derived from the expression level of the biomarkers in the tumor tissue $(\mathrm{T})$ compared with that of the normal thyroid tissue $(\mathrm{N})$. All the samples were analyzed in triplicate.

\section{Risk classification \& outcome variables used for participants in independent study}

Patients were classified as to whether they were at low, intermediate or high risk for recurrence based on the 2016 ATA risk stratification system [5].

Because of the small sample size, disease status was also consolidated into three binary outcome variables (initial metastasis, baseline status and longitudinal status) (Figure 2) that were examined in relation to the ITGA2, SYT12 and $C D H 3$ mRNA biomarkers.

\section{Initial metastasis}

The extent of metastases at initial presentation was assessed using information from the surgical pathology and results of initial therapy with RAI. This variable was dichotomized as no locoregional or distant metastasis (controls) versus either locoregional or distance metastasis (cases). 


\section{Baseline status}

Patients were assessed at approximately 2 months after their initial treatment with surgery and radioactive iodine therapy for evidence of disease. Patients who showed no evidence of disease after initial treatment were the controls, and the cases included patients with remaining biochemical disease, nodal disease or other metastases (e.g., pulmonary or skeletal) after initial treatment.

\section{Longitudinal status}

This variable, indicating whether a patient had progression or regression of disease, was generated from the annual evaluations of disease status over the 4- to 5-year follow-up. The regressors included patients with no evidence of disease at the baseline assessment who remained nondiseased over time; and patients with evidence of disease at the baseline who regressed to nondiseased. The progressors included patients with no evidence of disease at the baseline who progressed to diseased; and patients with evidence of disease at the baseline whose disease further progressed or remained stable.

\section{Statistical analysis for independent study}

Summary statistics were tabulated for the binary disease outcomes and biomarkers. A scatterplot matrix visualized the correlation structure of the three biomarkers. Using a continuous scale for the biomarkers, a receiver operating characteristic (ROC) curve was plotted for each of the biomarkers and outcomes. As a measure of the diagnostic/prognostic accuracy, the area under ROC curve (AUC) was calculated, and tested to determine if the AUC was greater than 0.5 using the Wilcoxon rank sum test. The significance level was 0.05 .

To study the sensitivity ( $\mathrm{Se}$ ) and specificity ( $\mathrm{Sp}$ ) of the biomarkers, we dichotomized them using prespecified cut-off points: ITGA2, SYT12 and CDH3 mRNA were positive if their fold expression was greater than or equal to 2, 4 and 10, respectively. Se and Sp were calculated, as well as their exact 95\% CI. As Se or Sp cannot be examined alone, but are better viewed together as a tradeoff between false-positive and false-negative errors, we also calculated Youden's index [12]. This is a summary measure of Se and Sp, defined as Se + Sp -1 . A larger Youden's index represents better accuracy of a binary test.

A combination of the several biomarkers can often improve diagnosis with, for example, multiple positive biomarkers being a strong indication of disease. Therefore, we counted the number of positive biomarkers (ranging from 0 to 3) for each patient, and then defined a 'positive result' of the combined markers in two ways: two or more markers positive and all three markers positive. The Se, Sp and Youden's index of the combinations were evaluated.

Since ATA risk categories are a commonly used clinical assessment of the possibility of recurrence, we also estimated the accuracy of ATA risk stratification in predicting the longitudinal outcome. After dichotomizing ATA risk as low versus intermediate/high, we calculated its Se, Sp and Youden's index for predicting the longitudinal disease outcome, and qualitatively compared them with the accuracy measures of the three biomarkers.

All the statistical analyses were conducted in $\mathrm{R}$ version 3.1.3.

\section{Results}

\section{Characteristics \& outcomes of the PTC patient cohort}

The characteristics of the cohort are described in section $2 \mathrm{~b}$ of the 'Materials $\&$ methods'. Our study cohort received care at MedStar Washington Hospital Center from either their endocrinologist alone, or from their endocrinologist and other specialists. A total of $76 \%$ of patients had four full years of follow-up; the remaining $24 \%$ of patients had 5 years of follow-up (Table 3 and Table 4). A total of $66 \%$ of patients had locoregional or distant metastases at presentation, 34\% still had evidence of disease after their initial treatment (Table 3). At the end of 4 years of follow-up, 30/38 (79\%) of patients had no evidence of PTC, whereas 8/38 (21\%) had residual disease that was stable in five patients and progressive in three patients. The eight patients that had residual disease had disease present in cervical lymph nodes, the lungs, the skeleton and the liver (Table 4). No patient died during the 4-5 years of follow-up. The three outcome variables of initial metastases, baseline status and longitudinal status and ATA risk category are summarized as percentages in Table 5 and Table 6, respectively. 


\section{Table 3. Longitudinal follow-up of the patients in the prospective study: DTC status.}

\section{Category}

DTC status ${ }^{\dagger}$

Baseline status

1-year follow-up

2-year follow-up

3-year follow-up

4-year follow-up

5-year follow-up

$\ddagger$ Baseline disease status after initial treatment was assessed based on a combination of surgical pathology, results of postoperative radioiodine scanning and postoperative thyroglobulin measurement at approximately 2 months after surgery.

DTC: Differentiated thyroid cancer.

\section{Number of patients in each category}

\begin{tabular}{lllll}
$\begin{array}{l}\text { Biochemical disease } \\
\text { only }\end{array}$ & $\begin{array}{l}\text { Residual structural } \\
\text { disease }\end{array}$ & Regression of disease & Stable disease & Progression of disease \\
\hline 4 & 9 & N/A & N/A & N/A \\
\hline 2 & 10 & 2 & 9 & 2 \\
\hline 1 & 8 & 2 & 6 & 1 \\
\hline 0 & 7 & 0 & 5 & 3 \\
\hline 0 & 8 & 0 & 2 & 1 \\
\hline 0 & 3 & 2 & 3 \\
$=9$ for fifth year of follow-up, may be more than one disease status per patient. &
\end{tabular}

\section{Table 4. Longitudinal follow-up of the patients in the prospective study: disease location.}

Number of patients in each category

${ }^{\dagger} \mathrm{n}=38$ patients for initial status and years $1-4, n=9$ for fifth year of follow-up, may be more than one disease location per patient.

${ }^{\ddagger}$ Baseline disease status after initial treatment was assessed based on a combination of surgical pathology, results of postoperative radioiodine scanning and postoperative thyroglobulin

\section{Table 5. Thyroid cancer status for patients in the prospective study.}

\section{Category}

Disease location

Baseline status

1-year follow-up

2-year follow-up

3-year follow-up

4-year follow-up

5-year follow-up measurement at approximately 2 months after surgery.

\section{Disease status}

\section{Initial metastasis}

No

Yes

\section{Baseline status}

No evidence of disease

None known
29
28
28
29
30
6

Pulmonary Skeletal

4

4

4

4

Summary statistics using count (percentage)

$$
13(34.2 \%)
$$

$25(65.8 \%)$

$25(65.8 \%)$

$13(34.2 \%)$

$30(78.9 \%)$

$8(21.1 \%)$

\section{Longitudinal status (Fourth year)}

Regression

$8(21.1 \%)$

$\dagger$ Initial metastasis $=0$ if none; 1 if metastasis is documented.

${ }^{\ddagger}$ Baseline status $=0$ if no disease, 1 if any disease still present.

$\S$ Longitudinal status $=0$ if initial status $=1$ and regressed to NED, or initial status $=0$ and stayed at NED (those who recovered) $;=1$ if initial status $=1$ and remained stable $/$ progressed, or initial status $=0$ and progressed to diseased (those who did not recover)

NED: No evidence of disease.

\section{Table 6. ATA risk category for patients in the prospective study.}

\section{ATA risk category}

Low

Intermediate

High

ATA: American Thyroid Association.
Summary statistics using count (percentage)

$18(47.4 \%)$

$16(42.1 \%)$

$4(10.5 \%)$ 
mRNA expression studies

Total RNA quality

High-quality RNA was prepared from tissue samples of patients, as reported in the 'Material \& methods'. The RINs (RNA integrity) were between $9.2 \pm 0.5$; the ratio of the absorbance at 260 and $280 \mathrm{~nm}$ was $1.98 \pm 0.06$ for all samples.

\section{Expression of ITGA2, SYT12 \& CDH3 mRNA in PTC}

As previously described, we computationally selected 15 genes (IPCEF1, FN1, ITGA2, TPO, SYT12, GPM6A, DIO1, CRABP1, STRA6, TFF3, TM7SF4, OTOS, CDH3, DTX4, TACSTD2) that were differentially regulated in thyroid tumors. After real-time PCR screening analysis of these 15 genes in the exploratory PTC tissue samples (training set), we found three genes (ITGA2, SYT12 and CDH3) that had significant differential expression in PTC tissues compared with normal thyroid tissues and in aggressive tumors compared with more indolent ones. Therefore, we focused on these transcripts that were highly expressed in the PTC training set in order to potentially improve our test accuracy in this independent study. The expression of these three genes was therefore determined in our prospective cohort of 38 patients with PTC. The $\log 2$ transformed fold changes in these genes in the tumor tissue of each patient are shown in Table 7, with the fold changes in TPO and CRABP1 shown for comparison. The median change in expression was 5.2-fold (range: 0.19- to 71-fold; $\mathrm{p}<0.001$ ), eightfold (range: 0.1- to 507-fold; $\mathrm{p}<0.003$ ) and 15-fold (range: 0.1- to 323-fold; $\mathrm{p}<0.001$ ) for ITGA2, SYT12 and CDH3, respectively. Fold change cut-off levels with the optimum diagnostic efficiency derived from the ROC curves were two for ITGA2, four for SYT12 and ten for CDH3.

\section{Accuracy of the biomarkers}

The biomarker data are summarized in Table 8, with percentages reported for categorical variables and mean (SD) for continuous variables. The $\log 2$ transformed fold change in each of the biomarkers, and the percentage of patients who had $0-3$ positive biomarkers is shown.

The three markers were all positively correlated with each other (Figure 3), with correlation coefficients between 0.60 and 0.69. Figure 4A-C show plotted area under the ROC curves for ITGA2, SYT12 and CDH3 mRNA in association with the three outcomes: initial metastasis, baseline status and longitudinal status. Table 9 shows the area under these ROC curves (AUC) with the 95\% CI. SYT12 had the highest diagnostic accuracy for all three outcomes, with AUCs ranging from 0.671 to 0.746 (p-values: 0.017-0.045). The Wilcoxon rank sum test suggested that the AUCs for SYT12 were significantly larger than 0.5 . CDH3 had borderline significant AUCs between 0.637 and 0.692 , and p-values ranging from 0.052 to 0.089 . ITGA2 only had marginal significant for prediction of longitudinal disease progression. The combination of all three biomarkers was not superior to the performance of SYT12 alone.

Table 10 shows the diagnostic accuracy of the biomarkers in predicting the dichotomized outcomes, and reports their Se and Sp with the exact 95\% CI. SYT12 had the best performance among the three markers for diagnosing initial metastasis and for predicting disease progression over time, with Youden's indices of 0.26 and 0.47, respectively. ITGA2 had a Youden's index of 0.24 in predicting baseline residual disease. Also shown in Table 10, is the performance of the combined biomarkers based on the number of positive markers. Using a cut-off point of 2 or more positive biomarkers, the diagnostic accuracy was the highest for baseline and longitudinal status, with a Youden's index of 0.29 and 0.43 , respectively. Using all three positive biomarkers the accuracy for predicting initial metastasis was similar to that of SYT12 alone, with a Youden's index of 0.26.

ATA risk category was also used to predict longitudinal outcomes and this had an Se of 88\%, an Sp of 57\% and a Youden's index of 0.44 . When combined with two or more positive markers, the Se was unchanged, but the Sp improved to $70 \%$, with a Youden's index of 0.57 . When combined with all three markers being positive, Se declined to $75 \%$, although Sp increased to 77\%, with a Youden's index of 0.52 . However, the best performance was obtained using a combination of ATA risk category and SYT12 with an Se of 88\%, an Sp of 73\% and a Youden's index of 0.61 .

\section{Discussion}

In this research study, we interrogated 15 mRNA that were associated with thyroid tumors and had differential expression in a high-throughput expression dataset. We performed an initial qPCR screening to further determine 
Table 7. Fold change in mRNA expression in tumor tissue compared with matched normal tissue, log 2 transformed and normalized to 18s rRNA for each patient in the prospective cohort.

\begin{tabular}{|c|c|c|c|c|c|}
\hline \multirow[t]{2}{*}{ Patient \# } & \multicolumn{5}{|c|}{ Genes } \\
\hline & SYT12 & ITGA2 & $\mathrm{CDH} 3$ & TPO & CRABP1 \\
\hline 1 & 179.15 & 3.64 & 29.45 & 0.02 & 0.41 \\
\hline 2 & 19.03 & 4.74 & 15.67 & 0.25 & 0.26 \\
\hline 3 & 4.58 & 2.96 & 14.72 & 0.08 & 0.09 \\
\hline 4 & 7.57 & 2.00 & 1.66 & 3.41 & 1.99 \\
\hline 5 & 4.86 & 9.51 & 2.48 & 0.03 & 0.00 \\
\hline 6 & 10.52 & 7.70 & 1.20 & 0.56 & 0.23 \\
\hline 7 & 0.06 & 0.19 & 3.11 & 0.67 & 0.57 \\
\hline 8 & 48.50 & 5.66 & 49.69 & 0.13 & 0.08 \\
\hline 9 & 20.32 & 3.51 & 18.96 & 0.07 & 0.03 \\
\hline 10 & 8.00 & 13.88 & 72.00 & 4.89 & 1.31 \\
\hline 11 & 374.81 & 28.94 & 51.80 & 0.13 & 0.27 \\
\hline 12 & 1.66 & 3.33 & 1.99 & 0.53 & 1.63 \\
\hline 13 & 16.91 & 54.00 & 93.70 & 0.01 & 0.12 \\
\hline 14 & 47.84 & 7.24 & 54.57 & 0.02 & 0.01 \\
\hline 15 & 48.17 & 16.97 & 92.09 & 0.01 & 0.02 \\
\hline 16 & 2.43 & 2.58 & 13.69 & 0.36 & 0.02 \\
\hline 17 & 2.32 & 0.69 & 3.64 & 0.30 & 0.46 \\
\hline 18 & 506.70 & 27.38 & 100.78 & 0.13 & 0.03 \\
\hline 19 & 63.78 & 19.56 & 67.88 & 0.00 & 0.00 \\
\hline 20 & 137.19 & 17.94 & 86.52 & 1.93 & 0.62 \\
\hline 21 & 1.02 & 1.78 & 3.73 & 0.55 & 0.47 \\
\hline 22 & 203.66 & 36.13 & 70.77 & 0.05 & 0.09 \\
\hline 23 & 1.01 & 1.50 & 2.21 & 1.60 & 0.92 \\
\hline 24 & 10.23 & 9.68 & 14.98 & 0.03 & 0.01 \\
\hline 25 & 58.28 & 42.81 & 314.08 & 0.22 & 0.26 \\
\hline 26 & 81.01 & 28.84 & 17.63 & 0.03 & 0.01 \\
\hline 27 & 69.31 & 50.04 & 116.97 & 0.68 & 0.30 \\
\hline 28 & 0.67 & 0.72 & 0.52 & 0.00 & 0.55 \\
\hline 29 & 1.11 & 0.99 & 12.08 & 0.51 & 0.24 \\
\hline 30 & 74.80 & 70.77 & 160.34 & 1.45 & 0.32 \\
\hline 31 & 0.45 & 1.32 & 1.52 & 15.51 & 1.97 \\
\hline 32 & 0.30 & 11.55 & 4.44 & 3.05 & 2.32 \\
\hline 33 & 0.35 & 9.03 & 1.81 & 3.04 & 66.26 \\
\hline 34 & 0.30 & 3.19 & 32.56 & 2.15 & 0.27 \\
\hline 35 & 28.64 & 5.22 & 322.91 & 0.01 & 0.01 \\
\hline 36 & 1.45 & 1.51 & 0.10 & 0.00 & 1.56 \\
\hline 37 & 0.84 & 2.71 & 4.17 & 2.44 & 2.11 \\
\hline 38 & 2.69 & 0.36 & 1.78 & 1.00 & 0.92 \\
\hline
\end{tabular}

how many of these 15 genes would be a useful prognostic biomarker for PTC, and found that ITGA2, SYT12 and $\mathrm{CDH} 3$ had significant differential expression in specimens from a tissue bank.

Biomarkers were then validated in resected thyroid tumors in a prospective study. Our current results indicate that high levels of these three genes tend to be associated with presentation with metastases, presence of residual disease after initial treatment and short-term longitudinal outcomes. Of these, SYT12 had greatest predictive ability based on the ROC curve. When examining Se and Sp, SYT12 was also the single-best predictor of initial metastases and longitudinal outcome. However, ITGA2 or two or more positive markers provided the best $\mathrm{Se}$, Sp or performance for predicting residual disease after initial treatment. However, all of these markers had relatively low predictive value. The finding that SYT12 is the best of the three biomarkers for PTC appears to be a congruent with the 
Table 8. Biomarker summary statistics: median (interquartile range) for fold change in biomarkers after log 2

transformation and count (percentage) for number of biomarkers.

\begin{tabular}{|l|l|}
\hline Biomarkers & Summary statistics \\
\hline ITGA2 $^{\dagger}$ & $6.4(2.6-19.2)$ \\
\hline SYT12 $^{\ddagger}$ & $10.4(1.5-55.8)$ \\
\hline CDH3 & \\
\hline Number of positive markers & $16.7(3.2-71.7)$ \\
\hline 0 & $7(18.4 \%)$ \\
\hline 1 & $6(15.8 \%)$ \\
\hline 2 & $4(10.5 \%)$ \\
\hline 3 & $21(55.3 \%)$ \\
\hline
\end{tabular}

${ }^{\dagger}$ ITGA2 is positive if greater than 2 .

\$SYT12 is positive if greater than 4 .

${ }^{\S} \mathrm{CDH} 3$ is positive if greater than 10 .

Table 9. Area under ROC curves for biomarker prediction.

\begin{tabular}{|llll|}
\hline Biomarker & \multicolumn{2}{c}{ Area under ROC curves X 100 (95\% confidence interval) } \\
\hline ITGA2 & Initial metastasis ${ }^{\dagger}$ & Baseline status & Longitudinal status \\
\hline SYT12 & $62.5(42.4,82.5)$ & $61.2(42.0,80.5)$ & $66.7(48.4,84.9)$ \\
\hline CDH3 & $69.2(51.5,87.0)$ & $67.1(49.3,84.9)$ & $74.6(57.6,91.6)$ \\
\hline
\end{tabular}

Bold indicates the best performance.

$\dagger$ Initial metastasis = 0 if none; 1 if metastasis is documented.

${ }^{\ddagger}$ Baseline status $=0$ if no disease, 1 if any disease is still present.

$\S$ Longitudinal status $=0$ if initial status $=1$ and regressed to NED, or initial status $=0$ and stayed at NED (those who recovered); $=1$ if initial status $=1$ and remained stable/progressed,

or initial status $=0$ and progressed to diseased (those who did not recover).

NED: No evidence of disease; ROC: Receiver operating characteristic.

See Figure $4 \mathrm{~A}-\mathrm{C}$ for actual ROC curves.

recent finding that SYT12 is one of the genes in the 71-gene signature that distinguishes BRAF-like tumors from NRAS-like tumors [13]. In addition, $\mathrm{CDH} 3$ gene expression has been found to discriminate PTC from normal thyroid tissue [14], and ITGA2 gene expression has been associated with PTC and aggressiveness of PTC [15-17].

The SYT12 gene or synaptotagmin 12 (also known as synaptotagmin-related gene 1 or SRG1) encodes a family of proteins involved in the regulation of transmitter release in the nervous system. SYT12 is rapidly inducible by thyroid hormone and the timing of its expression is consistent with regulation by thyroid hormone during development [18]. SYT12 is likely a part of a cascade of gene activation induced by thyroid hormone that is critical for CNS organization and development [19]. Regulation by thyroid hormone is not a feature common to all members of the synaptotagmin family, suggesting that SYT12 may have unique functions among the synaptotagmins. As mentioned above, the SYT12 gene is also associated with BRAF-positive tumors [13].

The ITGA2 gene encodes alpha-2 integrin, a membrane glycoprotein known as GP Ia, which is expressed in a variety of cell types. Integrins are transmembrane adhesion molecules that mediate cell-cell and cell-extracellular matrix attachment. As a consequence, integrins regulate cell growth, proliferation, migration and apoptosis and, thus, have a potential role in tumor progression and metastasis. ITGA2 has been shown to be differentially overexpressed in PTC compared with benign thyroid tissue $[15,17]$ and in aggressive compared with nonaggressive PTC [16]. The effect of ITGA2 seems to be mediated via abherent expression of miRNAs such as miR-107, miR-103 and miR-195. Cadherins, such as $C D H 3$, are integral membrane glycoproteins responsible for calcium-dependent cell-cell adhesion. $\mathrm{CDH} 3$ was found to be helpful in discriminating PTC from normal tissue when included in a panel of 19 genes [14]. However, many other molecular profiling studies have failed to implicate the ITGA2, SYT12 and $\mathrm{CDH} 3$ genes in thyroid tumorigenesis [20-23].

Interestingly, the gene that we found to be the strongest prognosticator, $S Y T 12$, is one of the genes that is associated with tumors that carry a BRAF V600E mutation [13]. SYT12 is important in brain development and reduction in thyroid hormone levels leads to reduced SYT12 expression in the fetal brain. Given that low TSH concentrations (presumably accompanied by high thyroid hormone levels) are beneficial for thyroid cancer outcomes [2,3], it could be speculated that the negative impact of SYT12 is not mediated through high thyroid hormone levels. The worse 


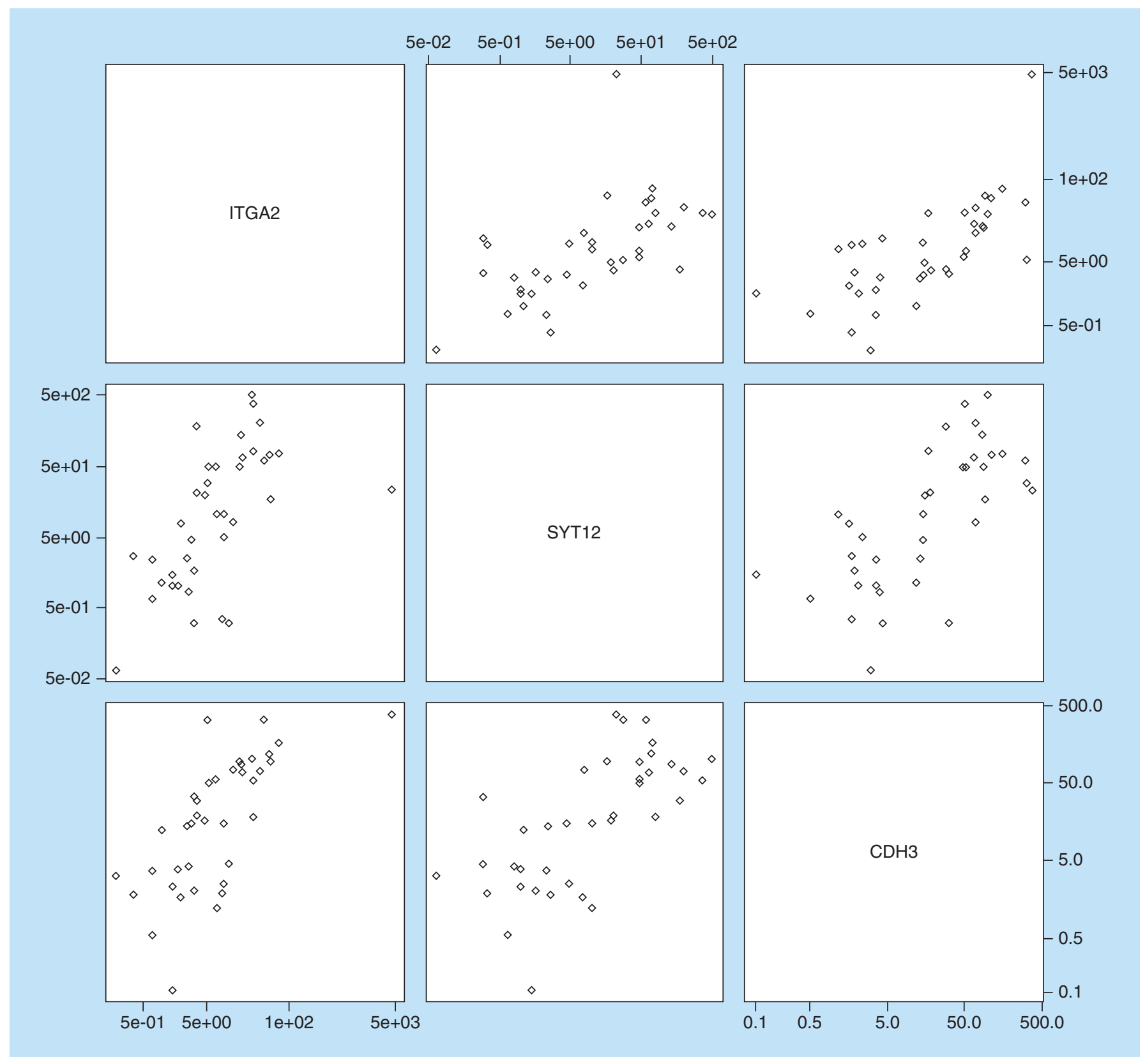

Figure 3. Scatterplot matrix for ITGA2, SYT12 and CDH3 visualizing the correlation structure of the three biomarkers using the relative fold change in log 2 scale in the marker in tumor tissue compared with normal thyroid tissue. Correlation coefficients are between 0.60 and 0.69 .

longitudinal outcomes associated with $S Y T 12$ could be associated with the BRAF mutation, or could be speculated to be associated with refractoriness to RAI therapy.

There are several limitations of our study. These include a small sample size in the independent cohort, a short duration of follow-up, conduct of the study prior to the publication of informative data regarding other gene sets and lack of $B R A F$ status for these tumors. The small sample size and resultant small number of outcomes necessitated dichotomized outcomes for the purposes of analysis. Ideally, longer than 4 years would be needed to assess disease progression in PTC. This study was approved in 2010 and conducted prior to the publication of databases such as the Cancer Genome Atlas, such that the comparison of these genes sets was not included in the study protocol. Furthermore, $B R A F$ status is not available for these tumors. In addition, Washington Hospital Center is a referral center for DTC and the distribution of disease, such as the high percentage of patients with 
(A)

ROC curve - metastasis

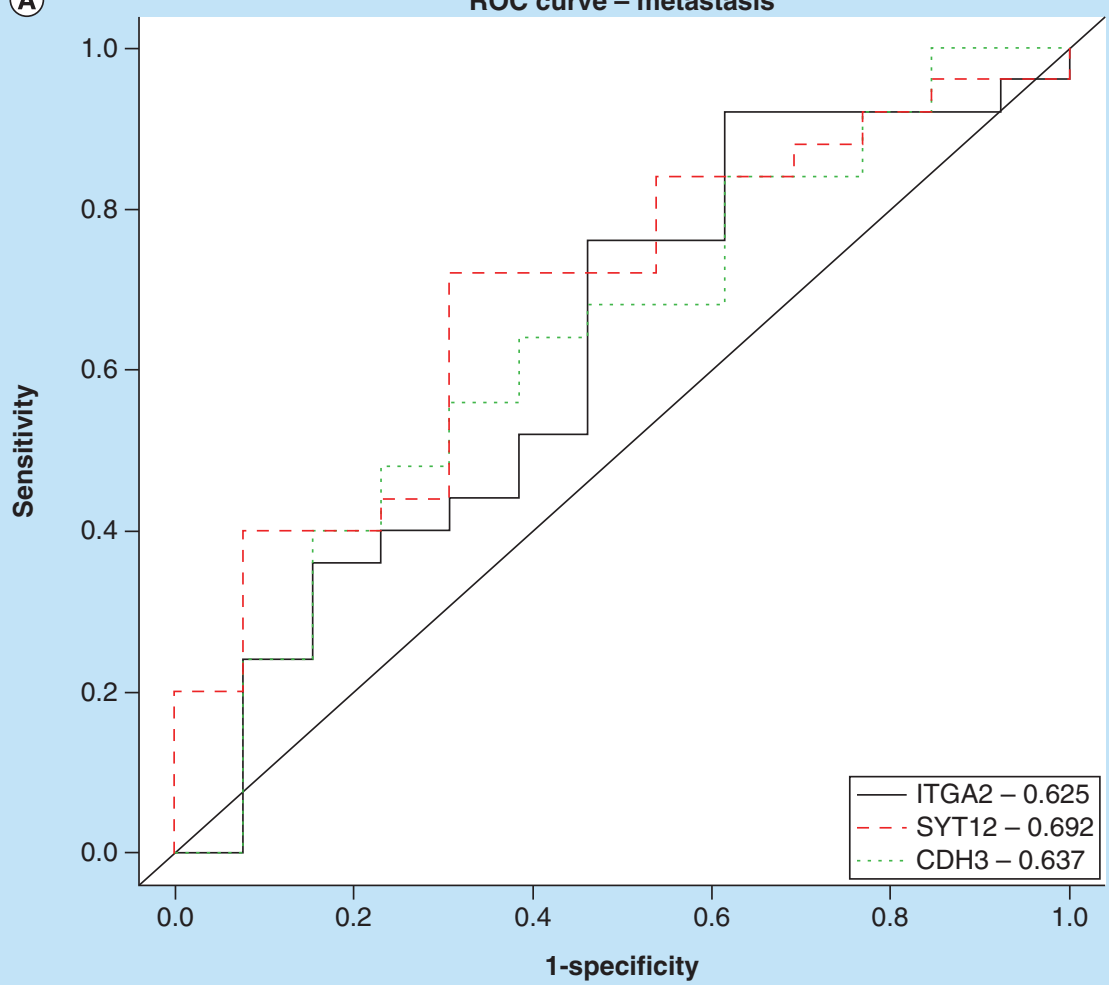

(B)

ROC curve: baseline assessment

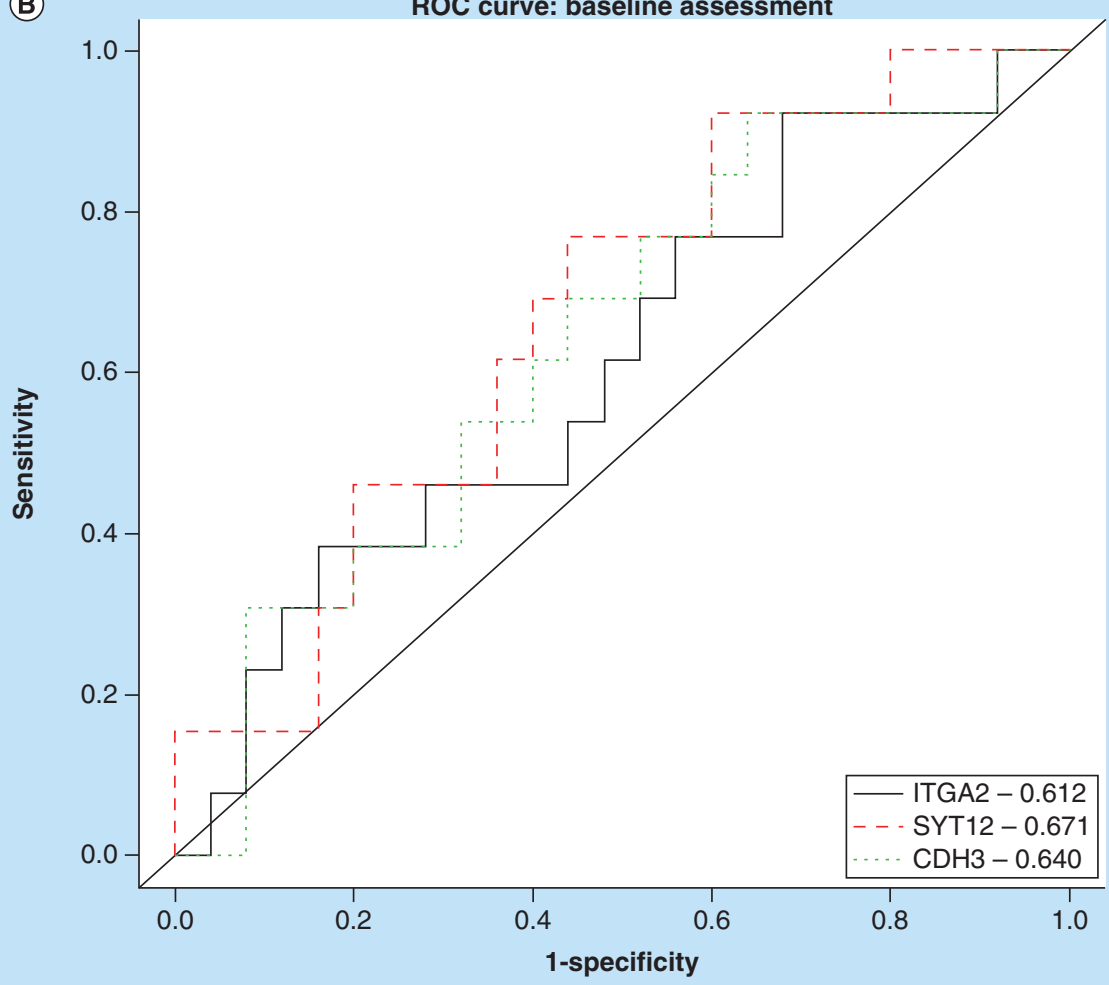

Figure 4. ROC curves for ITGA2, SYT12 and CDH3 as predictors of PTC outcomes. (A) Area under ROC curves for ITGA2, SYT12 and CDH3 demonstrating performance in diagnosing PTC metastasis at initial presentation (ROC values indicated within box). (B) Area under ROC curves for ITGA2, SYT12 and CDH3 demonstrating performance in predicting baseline status of residual disease after initial treatment (ROC values indicated within box). (C) Area under ROC curves for ITGA2, SYT12 and CDH3 demonstrating performance in predicting longitudinal status of disease progression after initial treatment (ROC values indicated within box).

PTC: Papillary thyroid cancer; ROC: Receiver operating characteristic. 
(C) ROC curve: longitudinal assessment

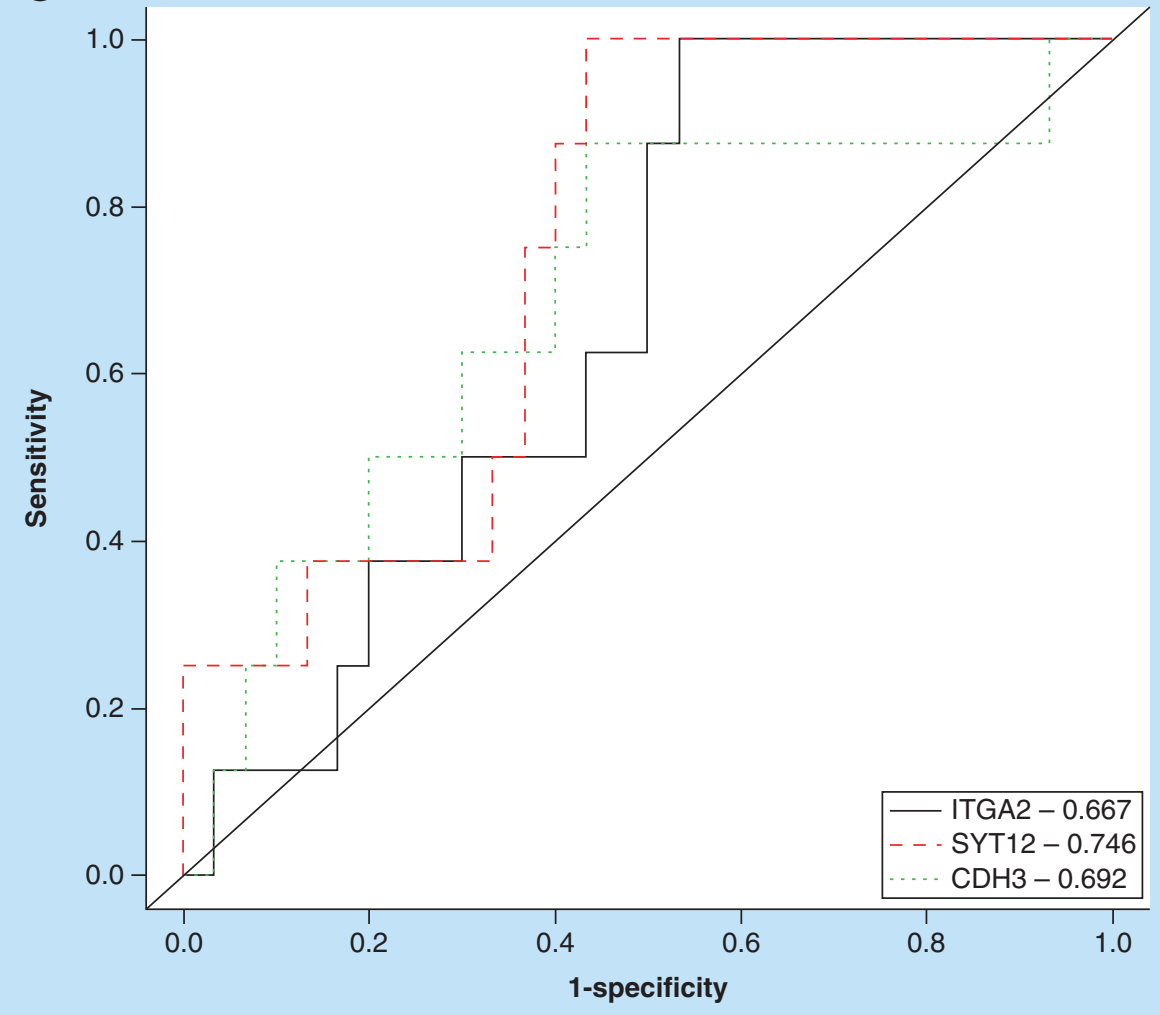

Figure 4. ROC curves for ITGA2, SYT12 and CDH3 as predictors of PTC outcomes (cont.). (A) Area under ROC curves for ITGA2, SYT12 and CDH3 demonstrating performance in diagnosing PTC metastasis at initial presentation (ROC values indicated within box). (B) Area under ROC curves for ITGA2, SYT12 and CDH3 demonstrating performance in predicting baseline status of residual disease after initial treatment (ROC values indicated within box). (C) Area under ROC curves for ITGA2, SYT12 and CDH3 demonstrating performance in predicting longitudinal status of disease progression after initial treatment (ROC values indicated within box).

PTC: Papillary thyroid cancer; ROC: Receiver operating characteristic.

extra-thyroidal spread of their tumor and distant metastases, may not be representative of the general population of patients with PTC. These limitations lead to data that are suggestive, but require validation in a larger dataset or cohort.

In summary, the biomarkers examined in this study may improve PTC risk stratification in several ways. They appear to have better performance in predicting longitudinal outcomes than features of initial presentation. SYT12 has the best accuracy as a single marker, and may be one of the genes conferring worse outcomes in $B R A F$-positive tumors. It has better Se and lower Sp than ATA risk stratification for longitudinal outcomes. However, the best performance was obtained by combining SYT12 with ATA risk stratification. SYT12 and ATA risk stratification did not have perfect concordance, again with SYT12 having better Se and ATA risk stratification having better Sp. This suggests that these markers may be reflecting different properties of the disease.

\section{Conclusion}

To our knowledge, ITGA2, SYT12 and CDH3 have not been prospectively investigated as biomarkers for predicting disease outcomes in patients with PTC. In this study, we recruited a cohort of patients with PTC and determined the levels of ITGA2, SYT12 and CDH3 mRNA in resected thyroid tumors. We examined the accuracy of these markers in predicting baseline and prospective disease status. The three computationally selected and experimentally validated biomarkers show some promising results that warrant further investigation in larger studies. 
Table 10. Sensitivity and specificity of the biomarkers in diagnosis and prediction of outcomes in the prospective cohort of patients.

\begin{tabular}{|c|c|c|c|}
\hline & Sensitivity $(95 \% \mathrm{CI})$ & Specificity $(95 \% \mathrm{Cl})$ & Youden's index \\
\hline \multicolumn{4}{|l|}{ Outcome: initial metastasis } \\
\hline ITGA2 & $0.84(0.64,0.95)$ & $0.38(0.14,0.68)$ & 0.22 \\
\hline SYT12 & $0.72(0.51,0.88)$ & $0.54(0.25,0.81)$ & 0.26 \\
\hline $\mathrm{CDH} 3^{\S}$ & $0.68(0.46,0.85)$ & $0.46(0.19,0.75)$ & 0.14 \\
\hline \# Positive markers $\geq 2$ & $0.72(0.51,0.88)$ & $0.46(0.19,0.75)$ & 0.18 \\
\hline \# Positive markers $=\mathbf{3}$ & $0.64(0.43,0.82)$ & $0.62(0.32,0.86)$ & 0.26 \\
\hline \multicolumn{4}{|l|}{ Outcome: baseline status } \\
\hline ITGA2 & $0.92(0.64,1.00)$ & $0.32(0.15,0.54)$ & 0.24 \\
\hline SYT12 & $0.77(0.46,0.95)$ & $0.44(0.24,0.65)$ & 0.21 \\
\hline $\mathrm{CDH} 3$ & $0.77(0.46,0.95)$ & $0.44(0.24,0.65)$ & 0.21 \\
\hline \# Positive markers $\geq 2$ & $0.85(0.55,0.98)$ & $0.44(0.24,0.65)$ & 0.29 \\
\hline \# Positive markers $=3$ & $0.69(0.39,0.91)$ & $0.52(0.31,0.72)$ & 0.21 \\
\hline \multicolumn{4}{|l|}{ Outcome: longitudinal status } \\
\hline ITGA2 & $1.00(0.52,1.00)$ & $0.30(0.15,0.49)$ & 0.30 \\
\hline SYT12 & $1.00(0.52,1.00)$ & $0.47(0.28,0.66)$ & 0.47 \\
\hline $\mathrm{CDH} 3$ & $0.88(0.47,1.00)$ & $0.43(0.25,0.63)$ & 0.31 \\
\hline \# Positive markers $\geq 2$ & $1.00(0.52,1.00)$ & $0.43(0.25,0.63)$ & 0.43 \\
\hline \# Positive markers $=3$ & $0.88(0.47,1.00)$ & $0.53(0.34,0.72)$ & 0.41 \\
\hline ATA risk (intermediate/high) & $0.88(0.47,1.00)$ & $0.57(0.37,0.75)$ & 0.44 \\
\hline $\begin{array}{l}\text { ATA risk (intermediate/high) and SYT12 } \\
\text { positive }\end{array}$ & $0.88(0.47,1.00)$ & $0.73(0.54,0.88)$ & 0.61 \\
\hline $\begin{array}{l}\text { ATA risk (intermediate/high) and \# } \\
\text { positive markers } \geq 2\end{array}$ & $0.88(0.47,1.00)$ & $0.70(0.51,0.85)$ & 0.57 \\
\hline $\begin{array}{l}\text { ATA risk (intermediate/high) and \# } \\
\text { positive markers }=3\end{array}$ & $0.75(0.35,0.97)$ & $0.77(0.58,0.90)$ & 0.52 \\
\hline \multicolumn{4}{|c|}{$\begin{array}{l}\text { †TGA2 is positive if greater than } 2 \text {. } \\
¥ \text { ॠST12 is positive if greater than } 4 \text {. } \\
\text { \$CDH3 is positive if greater than } 10 \text {. } \\
\text { Note: Exact confidence intervals were calculated due to small counts in the } 2 \text { by } 2 \text { table. } \\
\text { Bold indicates highest Youden's index for each outcome. } \\
\text { ATA: American Thyroid Association. }\end{array}$} \\
\hline
\end{tabular}

The demonstration that increased expression of these genes has a negative impact on PTC outcomes, which may lead to the future development of additional therapies for PTC. Furthermore, the methodology used in this study can be applied to other carcinomas.

\section{Future perspective}

Prognostication and selection of appropriate treatment are critical aspects of caring for patients with thyroid cancer. Tools to predict PTC outcomes are relatively imperfect and generally survival is more accurately predicted than recurrence. Treatment is often not selectively applied as the factors governing response to treatment are not fully understood. Use of genetic biomarkers, and perhaps among them SYT12 expression, may allow better prediction of outcomes and more targeted selection of treatment. As more genes involved in thyroid cancer are identified, the challenge will be to determine their role in cancer development and their interaction with each other. SYT12 appears to be one of the genes associated with the action of $B R A F$.

\section{Acknowledgements}

Contributions: J Jonklaas and YP Loh had overall responsibility for the entire design and conduct of the study; SRK Murthy was responsible for design and laboratory expertise; KD Burman, L Boyle, N Carrol and E Felger were responsible for study implementation; J Krishnan provided pathology expertise; D Liu provided statistical expertise; J Klubo-Gwiezdzinska was responsible for design and implementation. 
Summary points

- ITGA2, SYT12 and CDH3 had differential expression in papillary thyroid cancer (PTC) tumors compared with normal thyroid tissues.

- ITGA2, SYT12 and CDH3 generally had differential expression in PTC tumors that were more aggressive versus less aggressive in their presentation and progression.

- SYT12 predicted initial metastases (the extent of metastases at initial presentation) in a prospective PTC cohort (sensitivity [Se]: $72 \%$; specificity [Sp]: 54\%).

- Two or more positive biomarkers predicted baseline status (the presence or absence of disease after initial treatment) in a prospective PTC cohort (Se: $85 \%$; Sp: 44\%).

- SYT12 predicted longitudinal status (the presence or absence of disease during follow-up) in a prospective PTC cohort (Se: 100\%; Sp: 47\%).

- American Thyroid Association risk category had an Se of $88 \%$ and $\mathrm{Sp}$ of $57 \%$ for predicting longitudinal status in a prospective PTC cohort.

- SYT12 combined with ATA risk stratification best predicted longitudinal status in a prospective PTC cohort (Se: $88 \%$; Sp: 73\%).

Financial \& competing interests disclosure

The research of SRK Murthy, D Liu and YP Loh is supported by the Intramural Research Program of the National Institutes of Health $(\mathrm{NIH})$, Eunice Kennedy Shriver National Institute of Child Health and Human Development (NICHD). The authors have no other relevant affiliations or financial involvement with any organization or entity with a financial interest in or financial conflict with the subject matter or materials discussed in the manuscript apart from those disclosed.

No writing assistance was utilized in the production of this manuscript.

Ethical conduct of research

The authors state that they have obtained appropriate institutional review board approval or have followed the principles outlined in the Declaration of Helsinki for all human or animal experimental investigations. In addition, for investigations involving human subjects, informed consent has been obtained from the participants involved.

Open access

This work is licensed under the Creative Commons Attribution 4.0 License. To view a copy of this license, visit http:// creativecommons.org/licenses/by/4.0/

\section{References}

Papers of special note have been highlighted as: $\bullet$ of interest; $\bullet \bullet$ of considerable interest

1 Siegel RL, Miller KD, Jemal A. Cancer statistics, 2015. CA Cancer J. Clin. 65(1), 5-29 (2015).

2 Carhill AA, Litofsky DR, Ross DS et al. Long-term outcomes following therapy in differentiated thyroid carcinoma: NTCTCS Registry Analysis 1987-2012. J. Clin. Endocrinol. Metab. 100(9), 3270-3279 (2015).

-• Prospective database used to examine the long-term outcomes of differentiated thyroid cancer.

3 Jonklaas J, Sarlis NJ, Litofsky D et al. Outcomes of patients with differentiated thyroid carcinoma following initial therapy. Thyroid 16(12), 1229-1242 (2006).

4 Mcleod DS, Jonklaas J, Brierley JD et al. Reassessing the NTCTCS staging systems for differentiated thyroid cancer, including age at diagnosis. Thyroid 25(10), 1097-1105 (2015).

- Investigation of the effect of patient's age on the staging of papillary thyroid cancer.

5 Haugen BR, Alexander EK, Bible KC et al. 2015 American Thyroid Association Management Guidelines for adult patients with thyroid nodules and differentiated thyroid cancer: the American Thyroid Association Guidelines Task Force on Thyroid Nodules and Differentiated Thyroid Cancer. Thyroid 26(1), 1-133 (2016).

-. Current guidelines for the management of thyroid cancer from the American Thyroid Association.

6 Sherman SI, Brierley JD, Sperling M et al. Prospective multicenter study of thyroid carcinoma treatment: initial analysis of staging and outcome. National Thyroid Cancer Treatment Cooperative Study Registry Group. Cancer 83(5), 1012-1021 (1998).

7 Wong RM, Bresee C, Braunstein GD. Comparison with published systems of a new staging system for papillary and follicular thyroid carcinoma. Thyroid 23(5), 566-574 (2013). 
8 Tuttle RM, Tala H, Shah J et al. Estimating risk of recurrence in differentiated thyroid cancer after total thyroidectomy and radioactive iodine remnant ablation: using response to therapy variables to modify the initial risk estimates predicted by the new American Thyroid Association staging system. Thyroid 20(12), 1341-1349 (2010).

-• Thyroid cancer staging system that estimates recurrence rates based on response to therapy.

9 Braunstein. Thyroid cancer. In: Endocrine Updates. Melmed (Ed.). Springer, NY, USA (2012).

10 Xing M, Liu R, Liu X et al. BRAF V600E and TERT promoter mutations cooperatively identify the most aggressive papillary thyroid cancer with highest recurrence. J. Clin. Oncol. 32(25), 2718-2726 (2014).

11 Chruscik A, Lam AK. Clinical pathological impacts of microRNAs in papillary thyroid carcinoma: A crucial review. Exp. Mol. Pathol. 99(3), 393-398 (2015).

12 Zhou X, Obuchowski NA, Mcclish DK. Statistical Methods in Diagnostic Medicine (2nd Edition). Balding DJ, Cressie NAC, Fitzmaurice GM, Johnstone IM, Molenberghs G, Scott DW, Smith AFM, Tsay RS, Weisberg S (Eds). John Wiley \& Sons, Inc., Hoboken, NJ, USA (2011).

13 Cancer Genome Atlas Research N. Integrated genomic characterization of papillary thyroid carcinoma. Cell 159(3), 676-690 (2014).

-. Seminal article investigating the genome associated with papillary thyroid cancer.

14 Jarzab B, Wiench M, Fujarewicz Ket al. Gene expression profile of papillary thyroid cancer: sources of variability and diagnostic implications. Cancer Res. 65(4), 1587-1597 (2005).

15 Cong D, He M, Chen S, Liu X, Liu X, Sun H. Expression profiles of pivotal microRNAs and targets in thyroid papillary carcinoma: an analysis of The Cancer Genome Atlas. OncoTargets Ther. 8, 2271-2277 (2015).

16 Yang Z, Yuan Z, Fan Y, Deng X, Zheng Q. Integrated analyses of microRNA and mRNA expression profiles in aggressive papillary thyroid carcinoma. Mol. Med. Rep. 8(5), 1353-1358 (2013).

17 Liu X, He M, Hou Y et al. Expression profiles of microRNAs and their target genes in papillary thyroid carcinoma. Oncol. Rep. 29(4), 1415-1420 (2013).

18 Thompson CC. Thyroid hormone-responsive genes in developing cerebellum include a novel synaptotagmin and a hairless homolog. J. Neurosci. 16(24), 7832-7840 (1996).

- Demonstrating the role of SYT12 during development.

19 Potter GB, Facchinetti F, Beaudoin GM, 3rd, Thompson CC. Neuronal expression of synaptotagmin-related gene 1 is regulated by thyroid hormone during cerebellar development. J. Neurosci. 21(12), 4373-4380 (2001).

20 Fujarewicz K, Jarzab M, Eszlinger M et al. A multi-gene approach to differentiate papillary thyroid carcinoma from benign lesions: gene selection using support vector machines with bootstrapping. Endocr. Relat. Cancer 14(3), 809-826 (2007).

21 Montero-Conde C, Martin-Campos JM, Lerma E et al. Molecular profiling related to poor prognosis in thyroid carcinoma. Combining gene expression data and biological information. Oncogene 27(11), 1554-1561 (2008).

22 Hamada A, Mankovskaya S, Saenko V et al. Diagnostic usefulness of PCR profiling of the differentially expressed marker genes in thyroid papillary carcinomas. Cancer Lett. 224(2), 289-301 (2005).

23 Nilubol N, Sukchotrat C, Zhang L, He M, Kebebew E. Molecular pathways associated with mortality in papillary thyroid cancer. Surgery 150(6), 1023-1031 (2011). 
